\title{
HERRAMIENTAS PARA LA EVALUACIÓN DE RIESGOS SOBRE EL AMBIENTE Y SALUD, POR LA DISPOSICIÓN FINAL DE RESIDUOS SÓLIDOS URBANOS
}

\author{
Paulina Rebeca CÁRDENAS-MORENO ${ }^{1}$, Fabián ROBLES-MARTÍNEZ ${ }^{1}$, \\ Francisco José COLOMER-MENDOZA² y Ana Belem PIÑA-GUZMÁN ${ }^{1 *}$
}

\author{
${ }^{1}$ Instituto Politécnico Nacional, Unidad Profesional Interdisciplinaria de Biotecnología, Av. Acueducto s/n, \\ Barrio La Laguna, Col. Ticomán, C.P. 07340 Ciudad de México, México \\ ${ }^{2}$ Universidad Jaume I, Ingeniería Mecánica y Construcción, Av. Vicente Sos Baynat sn., 12071, Castellón, \\ España \\ *Autor para correspondencia: apinag@ipn.mx
}

(Recibido mayo 2016; aceptado septiembre 2016)

Palabras clave: contaminantes, biogás, lixiviados, exposición laboral, relleno sanitario, tiradero a cielo abierto

\section{RESUMEN}

En el campo de la protección ambiental y de la salud pública y ocupacional, es necesario controlar, en la medida de lo posible, las fuentes de emisión de contaminantes ambientales y su impacto. Para tal fin, es indispensable realizar investigaciones multidisciplinarias que apoyen el desarrollo de políticas públicas basadas en evidencia, encaminadas al beneficio social y cuyo costo económico asociado pueda ser medido. La evaluación de riesgos (ER) es una herramienta que, aplicada al sector del manejo de residuos sólidos urbanos (RSU) y la operación de sitios de disposición final (SDF), puede ayudar a estimar cuantitativamente y a jerarquizar los riesgos ocasionados por esos sitios. La información que una ER proporciona, servirá para apoyar la elaboración de medidas normativas de prevención de riesgos, así como en la toma de decisiones de control ambiental, a través del suministro de la mejor información científica disponible a las agencias normativas y entidades de gobierno. Los estudios de ER por la operación de un SDF de RSU con fines de investigación epidemiológica deben incluir a la población ocupacionalmente expuesta como la primera en estar en contacto con los contaminantes, para la identificación de rutas de exposición completas, la estimación y la medición de la exposición real a contaminantes, o bien, la elaboración de modelos de la exposición sobre la base de los datos existentes, avaladas por un equipo multidisciplinario de expertos en dichos sectores. Este trabajo expone las condiciones para la realización de cualquier estudio de ER al ambiente y a la salud humana por SDF de RSU, así como algunos instrumentos computacionales existentes que podrían ayudar a completar el procedimiento.

Key words: pollutants, biogas, leachate, occupational exposure, landfill, dump sites

\begin{abstract}
In the field of environmental protection and public health, it is necessary to control, to the extent possible, emission sources of environmental pollutants and their impact. To this purpose it is essential to carry out multidisciplinary research to support the development of public policies evidence-based, aimed at social benefit and whose associated economic cost can be measured. Risk assessment (RA) is a tool that, applied to the handling of
\end{abstract}


municipal solid waste (MSW) and the operation of waste disposal sites (WDS) sector, can help to quantitatively estimate and prioritize the risk caused by such sites. The information that a RA provides will support the development of risk prevention normative measures, as well as in decision on environmental control through the delivery of the best scientific information available to regulatory agencies and government agencies. The RA studies by the operation of WDS valuables for epidemiological research should include: the people occupationally exposed as the first to be in contact with the contaminants for the identification of complete exposure pathways, the estimation and measurement of actual exposure to contaminants or, the exposure modeling on basis of existing data, supported by a multidisciplinary team of experts in these sectors. This paper exposes the key points for conducting any RA study of WDS on the environment and human health, as well as some existing computational tools that could help to complete the task.

\section{INTRODUCCIÓN}

La evaluación de riesgos (ER), también llamada evaluación de riesgos ambientales, es un procedimiento fundamental para la prevención de daños a la salud, ocasionados por la exposición a sustancias, materiales o situaciones peligrosas. Su objetivo primordial es estimar la probabilidad de que se produzca un daño (y su severidad) a la salud humana y para el ambiente por una actividad o exposición a una sustancia potencialmente dañina (SEDEMA 2006; Taracido-Trunk 2011) y con base en ello determinar si es tolerable el riesgo que corre una población por estar expuesta a determinados agentes presentes en el ambiente. Esta herramienta, que se encuentra en continuo desarrollo, es aplicable a los distintos sectores económicos y sociales (como la industria alimentaria, la industria del petróleo, la gestión de hospitales, entre otros) en los que se identifique, por un lado una fuente emisora de agentes físicos o químicos potencialmente dañinos y por otro, un receptor, para establecer una asociación entre la exposición y los efectos adversos al ambiente y la salud (Butt et al. 2008b). Ambos elementos (fuente de contaminantes y receptores humanos) se encuentran en el sector del manejo y tratamiento de residuos sólidos.

En México, la disposición de los residuos sólidos urbanos (RSU) está sujeta a la norma oficial mexicana NOM-083-SEMARNAT-2003 (SEMARNAT 2003), que clasifica a los sitios de disposición final (SDF) en: Rellenos sanitarios (RESA), sitios controlados y sitios no controlados o tiraderos a cielo abierto, de acuerdo a la infraestructura y condiciones de manejo y operación del sitio. El impacto producido por la generación, manejo y disposición de los RSU en los SDF, ha recibido mayor atención en las últimas dos décadas, debido a las posibles enfermedades, el deterioro ambiental y paisajístico, alta afluencia vehicular, ruido, polvo y emisiones de olores relacionados con la operación de SDF. Actualmente, en el $95 \%$ de los municipios y delegaciones de nuestro país, los RSU se recolectan y disponen en SDF, sin embargo, sólo al $5 \%$ de los residuos se les da algún tratamiento (INEGI 2012). Algunos de estos sitios se encuentran ubicados cerca de las cabeceras municipales; en algunos casos se tiene control sobre ellos, mientras que la mayoría de éstos opera de manera deficiente e insegura. Así mismo, éste sector representa una fuente de trabajo para miles de personas que laboraran en estos sitios de manera formal primordialmente en los RESAs, como de manera informal en los Sitios No Controlados.

El biogás y lixiviados, generados en los SDF como principales productos de la biodegradación de los RSU, pueden contener cantidades variables de compuestos dañinos al ambiente y la salud. El grupo poblacional más afectado por las emanaciones de los SDF son las miles de personas que viven en las cercanías y/o trabajan en los SDF. La mayor parte de los problemas de salud humana provienen del biogás, a partir de sus compuestos orgánicos volátiles (COV) no metánicos y de las emisiones de contaminantes peligrosos del aire, además varios olores se liberan durante las operaciones de disposición de RSU (Palmiotto et al. 2014).

Además, algunos procesos que se emplean en el tratamiento de RSU pueden generar compuestos tóxicos que afectan a los seres vivos, un ejemplo es la incineración inadecuada o quema incontrolada, donde se pueden liberar compuestos como dioxinas y furanos, entre otros, que son altamente tóxicos.

El objetivo de este trabajo es presentar los aspectos importantes para la realización de estudios de ER al ambiente y a la salud humana para SDF de RSU, así como algunas herramientas computacionales existentes que podrían ayudar a completar el procedimiento.

\section{UBICACIÓN ADECUADA DE SDF}

Los problemas relacionados con la ubicación de SDF para RSU son críticos en virtud de que las áreas para establecerlos son limitadas, además de que deben 
encontrarse alejadas de comunidades o poblaciones, así como de cuerpos de agua, zonas forestales y ríos que pudieran resultar afectados por las emanaciones (Sissinno et al. 2000).

Las comunidades ubicadas cerca de los SDF están directamente expuestas a diversos agentes químicos a través de la inhalación de biogás liberado durante la degradación de los residuos y de los compuestos liberados por la quema accidental o intencional de los residuos en los SDF, sobre todo en los sitios no controlados. El contacto dérmico y la ingestión de agua potable obtenida de pozos contaminados por lixiviados, la inhalación e ingestión de partículas de suelo contaminado o el consumo de productos de cosecha propia cultivados en suelos contaminados con lixiviados o regados con agua contaminada con lixiviados, son otras posibles vías de exposición a los productos químicos ahí encontrados (Palmiotto et al. 2014).

El uso de suelo y el análisis de la capacidad de carga territorial, tienen como objetivo identificar patrones espaciales adecuados para el futuro uso del suelo, de acuerdo a las limitaciones específicas, preferencias o predicciones de alguna actividad; por lo tanto, existe una necesidad urgente de enfoques que pueden asegurar racionalmente la toma de decisiones, reducir al mínimo los impactos ambientales y, al mismo tiempo, aumentar la posibilidad de evitar la oposición pública a la instalación de sitios indeseables como los SDF (Demesouka et al. 2014). De acuerdo con Del Pozo-Manrique (1991), los terrenos en los que se asienta un SDF y su substrato definen sus niveles de seguridad hidrogeológica a largo plazo, por lo que todos aquellos terrenos hidrogeológicamente vulnerables deben ser descartados. Para ello, se deben descartar los suelos muy permeables como los sedimentarios con importante permeabilidad intergranular, materiales karstificables (calizas, dolomitas, yesos) y rocas con permeabilidad por fisuración (ígneas y metamórficas principalmente). Además de considerar aspectos técnicos, las decisiones para la localización adecuada de SDF deben respetar la legislación y la normatividad del país.

Estudios realizados por Colomer-Mendoza et al. (2013a, 2013b) muestran una serie de factores ambientales que se ven afectados por la ubicación de un SDF y son analizados de acuerdo a la normatividad española. Por otro lado, Davoli et al. (2010) y Palmiotto et al. (2014) han realizado evaluaciones integradas de riesgo en zonas cercanas a un RESA de Italia, estimando la exposición crónica a componentes emitidos en los sitios y concluyendo que, a pesar de la liberación de contaminantes tóxicos y carcinogénicos, el riesgo estimado fue despreciable debido a varios factores como la distancia de las poblaciones y las bajas concentraciones a la que se encontraban los contaminantes en la atmósfera.

\section{PRODUCCIÓN DE EFLUENTES EN SDF}

La degradación de la materia orgánica de los RSU comienza desde el almacenamiento o transporte de estos, sin embargo, a partir del momento en que son depositados y enterrados en los SDF, bajo condiciones de anaerobiosis, se inicia una serie de procesos fisicoquímicos y biológicos que, dependiendo de la composición de los residuos y de las condiciones ambientales, determinarán la cantidad y calidad de los efluentes (lixiviados y biogás) (Colomer-Mendoza y Robles-Martínez 2011). Estas emanaciones provenientes de los SDF pueden contaminar agua, suelo y aire.

Lixiviados. El lixiviado es el líquido que resulta de la percolación del agua a través de los residuos, cargándose a su paso de contaminantes como microorganismos y sustancias químicas minerales y orgánicas disueltas y en suspensión. Todos estos contamiantes representan un posible riesgo a la salud humana y a los ecosistemas. Por su origen y su composición tan compleja, los lixiviados de los SDF requieren de un manejo y tratamiento adecuados. Los principales factores que contribuyen a las características y la cantidad de lixiviados producidos en un SDF son la entrada de agua, incluyendo lluvia efectiva (precipitación menos evapotranspiración), la infiltración de las aguas subterráneas y superficiales, la disposición de residuos líquidos, la naturaleza misma de los residuos, la geología del sitio y el almacenamiento de líquido de la superficie (Aziz et al. 2012).

Öman y Junestedt (2008) señalan que un gran número de componentes peligrosos, que no han sido identificados, están presentes en los lixiviados. Identificar este tipo de compuestos es difícil, debido a la compleja mezcla característica de los lixiviados, su generación y su biodisponibilidad (Isidori et al. 2003 y Robles-Martínez 2008). En su estudio, Öman y Junestedt (2008) reportan que entre los muchos compuestos detectados se encuentran los alifáticos halogenados, bencenos y bencenos alquilados, fenoles y fenoles alquilados, etoxilatos, hidrocarburos aromáticos policíclicos, bencenos clorados, fenoles clorados, bifenilos policlorados, dioxinas cloradas y furanos clorados, compuestos ignífugos o retardantes de flama polibromados, plaguicidas, compuestos orgánicos de estaño, metil-mercurio y metales pesados. La presencia de este gran número de compuestos peligrosos en los lixiviados de SDF, al resultar en un impacto ambiental significativo, los hace importantes para las futuras evaluaciones de riesgo de SDF y el desarrollo de métodos de tratamiento de lixiviados.

Los lixiviados de SDF activos y clausurados pueden ser la mayor fuente de contaminación de aguas subterráneas y superficiales, además de impactar en la microflora y fauna del sitio por su alto contenido de metales pesados, 
componentes orgánicos y transporte de diversos contaminantes (Isidori et al. 2003), por lo que es necesario realizar su caracterización para conocer el potencial contaminante y poder llevar a cabo tratamientos adecuados y disminuir el impacto de los lixiviados al medio (González et al. 2011). Sin embargo, Robles-Martínez et al. (2011) analizaron los lixiviados del RESA más grande de México y encontraron que las concentraciones de metales pesados no superaron los límites establecidos por la norma oficial mexicana NOM-052-SEMARNAT-2005 (SEMARNAT, 2005), de acuerdo a la cual los lixiviados son considerados como residuos peligrosos y constituyen un factor de riesgo sanitario.

Biogás. El biogás está formado principalmente por metano $\left(\mathrm{CH}_{4}\right)$ y dióxido de carbono $\left(\mathrm{CO}_{2}\right)$ pero distintos autores (Chiemchaisri y Visvanathan 2008; Kiss-Köfalusi y Encarnación-Aguilar 2006; Spokas et al. 2006; Themelis y Ulloa 2007) han reportado, además de estos dos compuestos, otros en menor concentración (Cuadro I). Los componentes traza o componentes orgánicos no metánicos incluyen COV y contaminantes de aire peligrosos (CAP) (Soltani-Ahmadi 2000).

\section{CUADRO I. COMPOSICIÓN TÍPICA DEL BIOGÁS PRO-} DUCIDO EN SDF

\begin{tabular}{lc}
\hline Componente & $\begin{array}{c}\text { Porcentaje en } \\
\text { volumen }\end{array}$ \\
\hline Metano $\left(\mathrm{CH}_{4}\right)$ & $50-60$ \\
Dióxido de carbono $\left(\mathrm{CO}_{2}\right)$ & $40-50$ \\
Nitrógeno $\left(\mathrm{N}_{2}\right)$ & $2-3$ \\
Sulfuro de hidrógeno $\left(\mathrm{H}_{2} \mathrm{~S}\right)$ & $1-2$ \\
Hidrógeno $\left(\mathrm{H}_{2}\right)$ & $<1$ \\
Oxígeno $\left(\mathrm{O}_{2}\right)$ & $<1$ \\
Monóxido de carbono $(\mathrm{CO})$ & Traza \\
Amoniaco $\left(\mathrm{NH}_{2}\right)$ & Traza \\
Hidrocarburos aromáticos y cíclicos & Traza \\
Compuestos orgánicos volátiles & Traza \\
\hline
\end{tabular}

\section{CONTAMINANTES QUE GENERAN PREOCUPACIÓN}

Algunos grupos de sustancias como los disolventes orgánicos, metales, hidrocarburos de petróleo totales, hidrocarburos aromáticos policíclicos, compuestos clorados como los bifenilos policlorados (PCBs), metano, dioxinas y furanos, asbesto, fármacos y plaguicidas, deben ser considerados en la priorización de contaminantes cuando se realiza una ER en SDF. Su importancia radica en que son contaminantes altamente tóxicos, persistentes, bioacumulables y tienen una gran movilidad ambiental. A estos grupos de contaminantes además deben agregarse aquellos que sean específicos de los SDF estudiados y que resulten de la caracterización del sitio, en la que deben considerarse las fuentes generadoras cercanas de residuos depositados (OMS 2001; Palmiotto et al. 2014).

Las sustancias liberadas a partir de estas fuentes podrían afectar potencialmente a las personas que viven cerca de los SDF a través de una serie de vías. Estas podrían incluir el transporte de sustancias en el aire, el movimiento de las sustancias en las aguas superficiales o subterráneas, o la deposición de sustancias en la tierra, y el posterior consumo por el ganado (Environment Agency 2010). La información toxicológica de algunos componentes del biogás y lixiviados se extrajo a partir de las hojas de seguridad de dichos compuestos (Cuadro II y Cuadro III). La información contenida en estos cuadros fue obtenida de los sitios web del Instituto Nacional para la Seguridad y Salud Ocupacional (NIOSH, por sus siglas en inglés) y de la Administración de Salud y Seguridad Ocupacional (OSHA, por sus siglas en inglés) (NIOSH 2012, OSHA 2012).

Los disolventes orgánicos son productos químicos de uso industrial y comercial, los cuales pueden provocar efectos muy graves sobre la salud humana. Los efectos de una exposición prolongada a algunos disolventes incluyen cáncer, lesiones en el sistema nervioso, lesiones en riñón, hígado, corazón o pulmones, anemias y leucemia, lesiones en la piel, daños al sistema reproductor y daños al sistema endócrino (Mancheño et al. 2008). Las exposiciones ocupacionales y ambientales a disolventes orgánicos han sido asociadas con efectos adversos a la salud humana, entre los cuales se incluyen neuropatías periféricas, con reacción a metil-n-butil cetona; encefalopatía crónica asociada a la exposición crónica a una amplia variedad de disolventes orgánicos (Rojas-Alegría 2011).

También se ha obtenido información sustancial sobre efectos tóxicos de los disolventes orgánicos. Según datos del Instituto Mexicano del Seguro Social (IMSS), en el año 2014 se presentaron 6,364 enfermedades del trabajo a nivel nacional, de las cuales 174 fueron a causa de afecciones respiratorias debidas a la inhalación de gases, humos, vapores y sustancias químicas de las cuales 64 se reportaron en la Ciudad de México y el EM.

Por otro lado, si hablamos de una exposición ambiental en donde existe la presencia de un número indeterminado de contaminantes y diversos fenómenos de exposición, es difícil establecer relaciones de causalidad entre las enfermedades y los contaminantes, a menos que se cuente con una metodología de ER que se fortalezca con la inclusión del mayor número de criterios y condicionantes, que permita considerar más factores involucrados en el riesgo. Además, a fin de reducir el grado de incertidumbre en los resultados, se debe contar preferentemente con 
CUADRO II. DATOS TOXICOLÓGICOS DE LOS PRINCIPALES COMPONENTES DEL BIOGÁS

\begin{tabular}{|c|c|c|c|c|c|c|}
\hline Comp. & LCLO (ppm) & CL50 & TWA* & $\begin{array}{l}\mathrm{IDLH}^{* *} \\
(\mathrm{ppm})\end{array}$ & $\begin{array}{l}\text { Rutas de } \\
\text { exposición }\end{array}$ & Síntomas o Efectos \\
\hline $\mathrm{CO}_{2}$ & $\begin{array}{l}90000 / 5 \mathrm{~min} \\
\text { En humanos }\end{array}$ & & $\begin{array}{c}5000 \mathrm{ppm} \\
(9000 \mathrm{mg} / \mathrm{m} 3)\end{array}$ & 40000 & $\begin{array}{l}\text { Inhalación, } \\
\text { y/o contacto } \\
\text { con la piel } \\
\text { y ojos (líqui- } \\
\text { do/sólido) }\end{array}$ & $\begin{array}{l}\text { Dolor de cabeza, mareos, nerviosis- } \\
\text { mo, parestesia; disnea (dificultad para } \\
\text { respirar); sudoración, malestar general } \\
\text { (sensación vaga de malestar); aumento de } \\
\text { la frecuencia cardíaca, gasto cardíaco, la } \\
\text { presión arterial; estado de coma; la asfixia; } \\
\text { convulsiones }\end{array}$ \\
\hline $\mathrm{CH}_{4}$ & & $\begin{array}{l}166.759 \mathrm{mg} / \mathrm{L} \\
\text { En peces }\end{array}$ & $1000 \mathrm{ppm}$ & & $\begin{array}{l}\text { La sustancia } \\
\text { se puede } \\
\text { absorber por } \\
\text { inhalación. }\end{array}$ & $\begin{array}{l}\text { Irrita los pulmones, los ojos, la piel y es } \\
\text { extremadamente inflamable }\end{array}$ \\
\hline $\mathrm{CO}$ & $\begin{array}{l}5000 / 5 \mathrm{~min} \\
\text { En humanos }\end{array}$ & $\begin{array}{l}1807 \mathrm{ppm} / 4 \mathrm{hrs} \\
\text { En ratas }\end{array}$ & $\begin{array}{c}50 \mathrm{ppm} \\
(55 \mathrm{mg} / \mathrm{m} 3) \\
\text { OSHA } \\
35 \mathrm{ppm} \\
(40 \mathrm{mg} / \mathrm{m} 3) \\
\mathrm{C} 200 \mathrm{ppm} \\
(229 \mathrm{mg} / \mathrm{m} 3) \\
\text { NIOSH }\end{array}$ & 1200 & $\begin{array}{l}\text { Inhalación, } \\
\text { y/o contacto } \\
\text { con la piel } \\
\text { y ojos (líqui- } \\
\text { do/sólido) }\end{array}$ & $\begin{array}{l}\text { Dolor de cabeza, taquipnea, náuseas, } \\
\text { lasitud (debilidad, agotamiento), mareos, } \\
\text { confusión, alucinaciones; cianosis; seg- } \\
\text { mento ST deprimido de electrocardiogra- } \\
\text { ma, angina, síncope }\end{array}$ \\
\hline $\mathrm{H}_{2} \mathrm{~S}$ & & & $\begin{array}{c}\text { C } 20 \mathrm{ppm} \\
50 \mathrm{ppm} \\
{[10-\text { minutos }} \\
\text { pico máximo }] \\
\text { OSHA } \\
\text { C } 10 \mathrm{ppm} \\
(15 \mathrm{mg} / \mathrm{m} 3) \\
{[10-\text { minutos }]} \\
\text { NIOSH }\end{array}$ & 100 & $\begin{array}{l}\text { La sustancia } \\
\text { se puede } \\
\text { absorber por } \\
\text { inhalación } \\
\text { y por inges- } \\
\text { tión. }\end{array}$ & $\begin{array}{l}\text { Irritación de ojos, del sistema respiratorio; } \\
\text { apnea, coma, convulsiones; conjuntivitis, } \\
\text { dolor ocular, lagrimeo (flujo de lágrimas), } \\
\text { fotofobia (intolerancia visual anormal a } \\
\text { la luz), vesiculación de la córnea; mareos, } \\
\text { dolor de cabeza, lasitud (debilidad, agota- } \\
\text { miento), irritabilidad, insomnio; trastornos } \\
\text { gastrointestinales. }\end{array}$ \\
\hline
\end{tabular}

*TWA, por sus siglas en inglés: tiempo promedio ponderado

**IDLH, por sus siglas en inglés: concentraciones inmediatamente peligrosas para la vida o la salud

información epidemiológica, de lo contrario, para evaluar la toxicidad (tanto aguda como crónica) de un compuesto, se suele recurrir a información de bioensayos en los que se ha expuesto de manera aguda a organismos vivos a diferentes concentraciones o dosis de la sustancia tóxica a probar. Estos estudios alternativos constituyen uno de los elementos de juicio más adecuado para la evaluación del riesgo potencial producido por un contaminante presente en el ambiente.

\section{EPIDEMIOLOGÍA AMBIENTAL}

Existen procesos patológicos que tienen una elevada mortalidad y morbilidad (la morbilidad se refiere al número de casos de enfermedad, no de muertes) y otros que, a pesar de ser frecuentes causan poca mortalidad (Vioque-López et al. 2011). En esta variabilidad influyen diferentes factores, pues la salud humana depende de la interacción entre factores genéticos y ambientales, y éstos últimos están determinados en gran medida por el nivel de desarrollo socioeconómico de la población, lo que genera grandes diferencias en los patrones de morbimortalidad entre distintas áreas del mundo. Numerosos estudios han demostrado que existe una relación entre el estado general de nuestra salud y la calidad de nuestro ambiente (Ballester-Diez et al. 2011), aunque establecer dichas asociaciones se dificulta dada la lentitud de acumulación de evidencias en poblaciones expuestas a contaminantes ambientales, así como la multiplicidad de contaminantes y la variabilidad 
A. B. Piña-Guzmán et al.

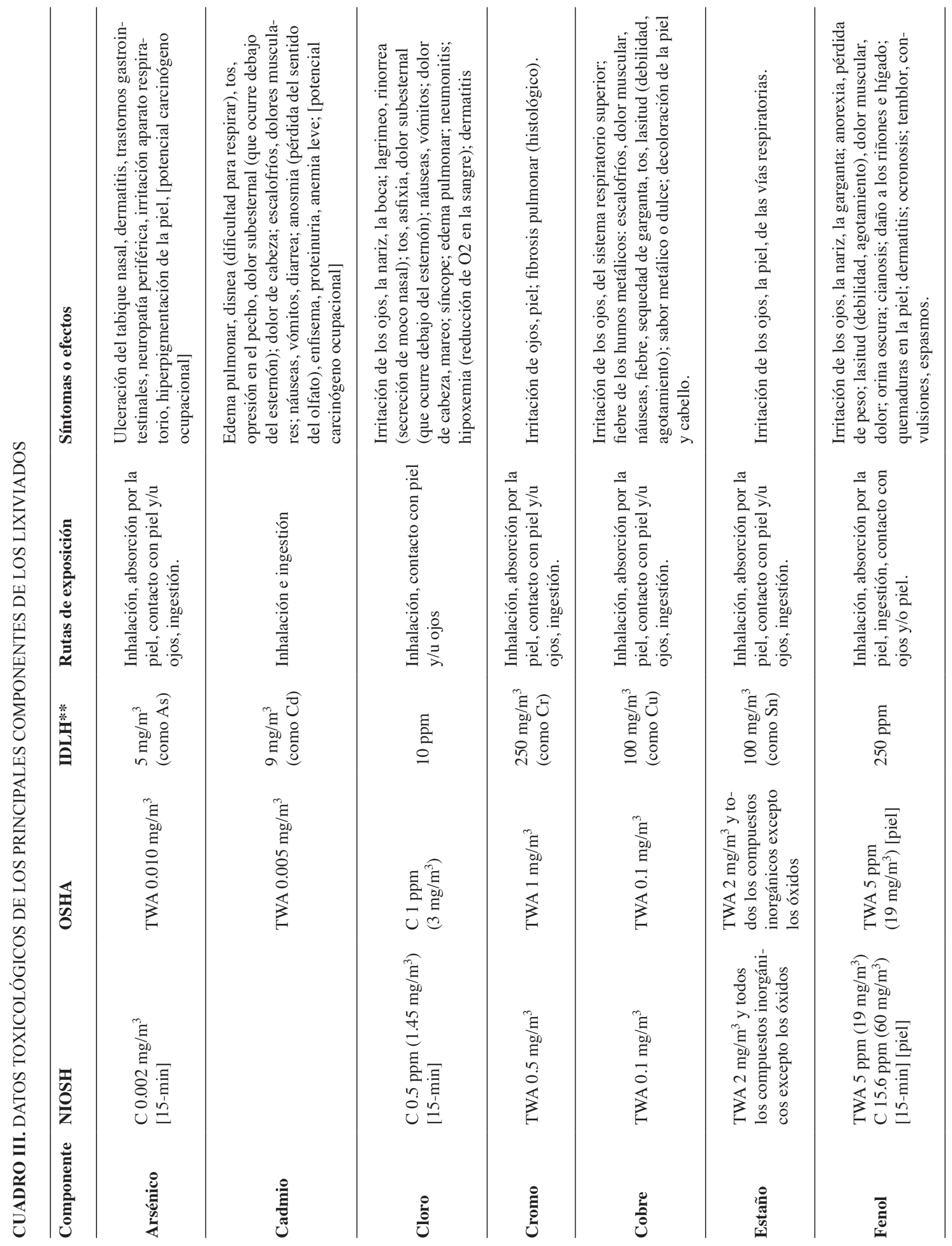


en las situaciones de exposición individual y de síntomas y trastornos de salud y el hecho de que síntomas específicos pueden corresponder a exposiciones diferentes.

Según el informe de la Organización Mundial de la Salud (OMS 2014), las infecciones de las vías respiratorias, cardiopatías, enfermedades pulmonares, anomalías congénitas, entre otras, se encuentran entre las primeras 20 causas de muerte a nivel mundial y en México. No debe descartarse la posibilidad de que en México algunas de estas estén relacionadas a la exposición a contaminantes ambientales y más específicamente a los provenientes del manejo inadecuado de residuos.

En los países en vías de desarrollo, predomina la disposición final de los residuos sólidos en el suelo. Es muy común que ésta se realice inadecuadamente y que los SDF no cumplan con los requisitos mínimos que aseguren la mitigación del impacto ambiental y que eviten la afectación de la salud pública. Entre los problemas más generalizados de la disposición final de los RSU, están la mezcla de residuos de diferentes fuentes y características, el recubrimiento deficiente, la pepena, la quema (accidental o intencional), así como, la carencia de infraestructura para el control de los lixiviados y biogás (Buenrostro et al. 2008). La consecuencia más inmediata de estos problemas es la alteración del agua, suelo y aire y de manera menos evidente, pero por demás preocupante, un efecto sobre la salud.

\section{EPIDEMIOLOGÍA ASOCIADA A SDF}

Con los años, se han planteado serios interrogantes acerca de la conveniencia de trabajar y vivir cerca de los SDF de RSU, por los posibles efectos sobre la salud. Estas preguntas han surgido de observaciones ocasionales de grupos en pueblos y aldeas cercanas a SDF con diagnósticos de alteraciones de salud. Estos grupos se investigaron mediante técnicas epidemiológicas para evaluar si la ocurrencia de los efectos sobre la salud podrían asociarse con fluctuaciones aleatorias o con la presencia de SDF o alguna otra causa, sin embargo los resultados siguen sin aseverar que ciertos padecimientos están asociados a los sitios (Environment Agency 2010).

Varios estudios epidemiológicos llevados a cabo por diversos autores (Vrijheid et al. 2002, Kloppenborg et al. 2005, Porta et al. 2009, Forastiere et al. 2011, Pukkala 2014, Triassi et al. 2015) para establecer el conocimiento sobre los efectos potenciales para la salud derivados de la exposición a los contaminantes de los SDF, concuerdan en que hay poca evidencia de una relación entre el riesgo de anomalías congénitas en la proximidad de las zonas aledañas a los SDF, pero sin una validación externa es difícil la interpretación de los métodos de evaluación potencial 
de riesgo. La clasificación de los SDF puede causar posibles errores y haber reducido la capacidad para detectar cualquier efecto de dosis-respuesta. Un nivel moderado de confianza es posible en áreas limitadas de conocimiento, lo que implica la necesidad de superar las limitaciones de los estudios actuales sobre la evaluación de la exposición y controlar los factores de confusión.

Las emisiones de contaminantes en los RESAs han sido estudiadas en detalle en los últimos años y la Environment Agency (2010) estableció que las principales fuentes de dichas emisiones son las siguientes:

- Los motores de los vehículos utilizados en las maniobras para disponer los RSU en las celdas.

- Los lixiviados producidos que sale del SDF.

- Cualquier gas generado por la degradación de los residuos, que no se colectó ni se trató.

Por otro lado, la noción de enfermedad profesional se origina en la necesidad de distinguir las enfermedades que afectan al conjunto de la población de aquellas que son el resultado directo del trabajo que realiza una persona, porque generan derechos y responsabilidades diferentes que las primeras (Sánchez-Aguilar 2013).

Entre los factores que determinan las enfermedades profesionales tenemos: variabilidad biológica del individuo, que explica que ante un mismo riesgo o condición patógena laboral, no todos enferman y los que enferman no lo hacen todos al mismo tiempo y con la misma intensidad; multicausalidad, es decir, una misma enfermedad puede tener distintas causas o factores laborales y extralaborales que actúan al mismo tiempo y que contribuye a su desencadenamiento; inespecificidad clínica, en la que la mayoría de las enfermedades profesionales no tiene un cuadro clínico específico que permita relacionar la sintomatología con un trabajo determinado; y condiciones de exposición, de acuerdo a lo cual un mismo agente puede presentar efectos nocivos diferentes según las circunstancias de exposición y vía de ingreso al organismo.

En la legislación sobre enfermedades profesionales, la introducción de la noción de daño previo a la enfermedad estimula la prevención porque implica una acción que la mayoría de las veces corresponde a la empresa directamente implicada y no sólo el organismo asegurador, que en la práctica sólo puede actuar después de la declaración de la enfermedad.

Lo anterior obliga a implementar programas de vigilancia de la salud, identificando los agentes y factores de riesgo, la población expuesta a ellos, la intensidad de la exposición y los indicadores que se utilizarán para la búsqueda del daño. Para ello, existe una ciencia que anticipa, reconoce y evalúa los riesgos perjudiciales en el ambiente laboral, así como desarrolla estrategias de prevención y control, con el objetivo de proteger y promover la salud y bienestar de los trabajadores, salvaguardando también la comunidad y el medio ambiente, que de acuerdo con la OMS se denomina salud ocupacional.

\section{EVALUACIÓN DE RIESGOS EN SITIOS DE DISPOSICIÓN FINAL}

La ER es un procedimiento esencial en la priorización de la protección al ambiente y a la salud humana. Sin embargo, los evaluadores deben comparar la gama completa de los riesgos, sobre una base sólida y consistente, considerando el mayor número posible de factores involucrados, para que la evaluación del riesgo se utilice como una ayuda más integrada en el proceso de toma de decisiones (Butt et al. 2013). En su estudio Butt et al. (2008a) reportaron una serie de trabajos de ER en SDF, señalando ciertas limitaciones metodológicas, pues los estudios realizados hasta ese momento, se enfocaban principalmente en el impacto de los lixiviados y sus componentes y carecían de automatización y precisión en el modelado de la movilidad de los contaminantes en los medios. En un estudio más reciente, Butt et al. (2013) señalan que pocas investigaciones se han realizado tomando en cuenta la necesidad, parámetros, requisitos y componentes del sitio a evaluar, en comparación con un sitio de referencia, sobre todo en el contexto de cómo, por qué y qué información debe ser cotejada con el fin de hacer evaluaciones de riesgo más adecuadamente integradas y completas.

La OMS expone que la evaluación del riesgo por la exposición a sustancias peligrosas liberadas por un SDF, también puede ser necesaria debido a una serie de razones, como la de evidenciar el grado de cumplimiento con lo dispuesto en la normatividad del país, atender las preocupaciones de la comunidad y desarrollar investigaciones epidemiológicas (OMS 2001).

El uso de métodos normalizados para la evaluación de la exposición permitiría la comparación entre los diferentes sitios de estudio, así como entre las exposiciones evaluadas por distintas investigaciones in situ. También puede facilitar el análisis combinado de resultados de estudios epidemiológicos y ayudar a la identificación de características específicas de los residuos sólidos que podrían estar asociadas con los resultados adversos para la salud.

Es importante tener conciencia de que la presencia de una fuente contaminante no es suficiente para causar un riesgo para la salud, tiene que existir una ruta de exposición completa; es decir, una fuente, medios ambientales y mecanismos de transporte, un punto de exposición, una vía de exposición y una población receptora (OMS 2001).

Para ello, la OMS propone una serie de pasos a seguir antes de realizar la evaluación de riesgos de un SDF. 
a) Realizar una caracterización del sitio a evaluar.

b) Realizar una caracterización de los receptores, mediante la descripción de la población circundante en riesgo de exposición potencial.

c) Identificar las rutas de exposición; es decir, aquellas vías que permiten que la población humana se vea expuesta.

d) Determinar la concentración de los contaminantes en los medios con los que los seres humanos podrían estar en contacto directo (como el suelo, el aire, los alimentos y el agua).

e) Realizar una estimación de la exposición a partir de los datos disponibles sobre concentraciones, mecanismos de ingestión y población en riesgo.

\section{METODOLOGÍAS}

Aunque existen diferentes metodologías para la ER a la salud, la más destacada es la que reporta la Agencia de Protección Ambiental de Estados Unidos (USEPA, por sus siglas en inglés) (Taracido-Trunk 2011), la cual consiste en un proceso de cuatro etapas (Fig. 1) para estimar el riesgo a la salud humana, como resultado de la exposición a un contaminante tóxico.

Derivada de la metodología anterior, la Metodología de Freeman (Martínez-Ponce de León 2007) está enfocada a los propósitos de la Higiene Industrial. Ortiz-Morales (2010) y Sánchez-Aguilar $(2013,2009)$ la definen como Diagnóstico Situacional (DS). Consta de las mismas etapas que la metodología anterior, sin embargo, recomiendan que no se aplique a población abierta (general), sino sólo a personal ocupacionalmente expuesto y que no se limite a los agentes químicos, sino a todos los riesgos conocidos (actos inseguros, condiciones inseguras y agentes contaminantes).
Ortiz-Morales (2010) señala que en el DS se realiza una investigación en tres niveles acerca de los efectos nocivos que pueden provocar los riesgos estudiados:

Histórico o retrospectivo: También se le conoce como epidemiología laboral. Consiste en indagar la incidencia y prevalencia de accidentes y enfermedades a consecuencia del trabajo, que han ocurrido en la organización, en un período y en un área laboral, específicos.

Aquí y ahora: Es el resultado del estudio actual sobre la siniestralidad ocurrida. Se complementa con las etapas previas sobre el reconocimiento del riesgo y evaluación de la exposición.

Peligrosidad intrínseca: Se refiere a lo que la literatura documenta sobre los efectos adversos de riesgos específicos, de acuerdo a reportes de estudios recientes, o de estudios pasados, pero aún vigentes.

La importancia de realizar ER en el personal laboral de SDF radica en el gran número de personas que laboran en estos sitios, tanto de manera formal como informal. Cabe resaltar que éste último grupo, principalmente se dedica la recolección de residuos o pepena y carece generalmente de capacitación y protección para el desarrollo de sus actividades. El DS para estos grupos laborales puede dar pie para contemplar los riesgos asociados por la emisión de contaminantes de SDF hacia las poblaciones aledañas a estos sitios.

La metodología del DS es aplicable al personal que labora en los SDF. Sus etapas se describen a continuación:

Identificación o reconocimiento del riesgo. Por definición, riesgo es la probabilidad de sufrir un daño por la exposición a un peligro, y peligro es la fuente del riesgo y se refiere a una sustancia o una acción que pueda causar daño. Esta primera etapa de la ER trata de identificar los efectos indeseables que una sustancia es intrínsecamente capaz de provocar. Para ello se utiliza información

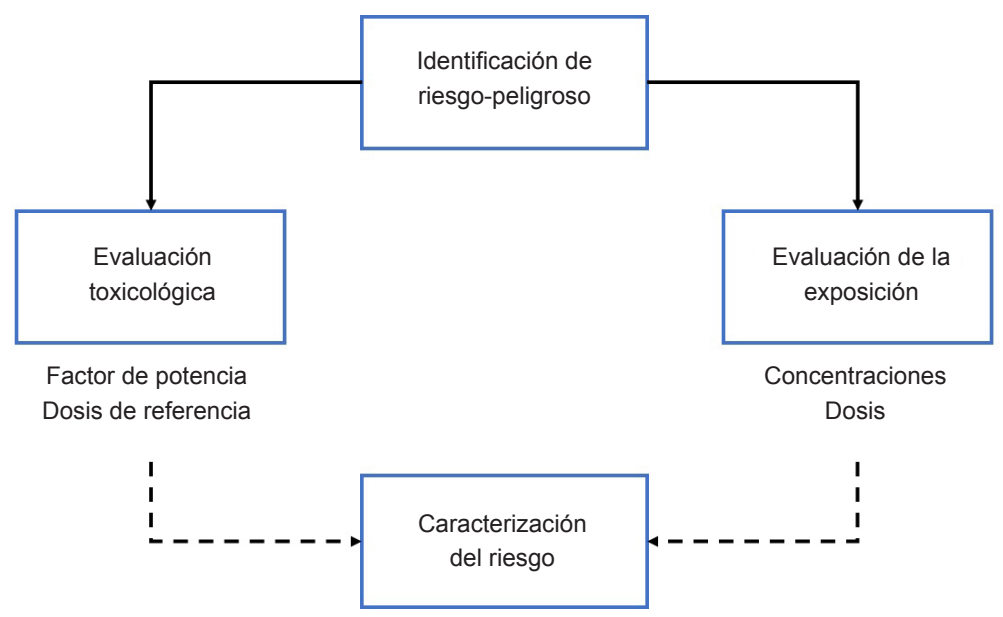

Fig. 1. Etapas de la evaluación de riesgos ambientales 
obtenida de estudios epidemiológicos, ensayos con animales, bases de datos de sistemas integrados de riesgo, etc. (Taracido-Trunk 2011).

En el DS se identifican los peligros y sus riesgos asociados, a través de la investigación y análisis del proceso productivo de la organización estudiada. Ello requiere de la descripción de cada etapa, cada puesto y cada actividad del proceso estudiado, utilizando como auxiliares un flujograma y un mapeo de riesgos.

Evaluación de la exposición. La OMS (2001) define a la exposición como la ocurrencia del contacto, en determinado tiempo y espacio, entre una persona y uno o más agentes biológicos, químicos o físicos. La exposición se describe mediante una relación que involucra la concentración de un agente ambiental en el medio de transporte (intensidad), la tasa de contacto en el organismo receptor, la frecuencia y la duración del evento. En el DS se mide el contacto directo del contaminante/peligro con el trabajador, a través de la frecuencia, duración e intensidad del evento.

Los métodos de evaluación cuantitativa de la exposición se pueden clasificar como métodos de evaluación directa (medida) o indirecta (estimada). Ambos métodos son independientes y complementarios. Cada uno se basa en diferentes tipos de datos y tiene diferentes ventajas y desventajas. Por lo tanto, es potencialmente útil emplear múltiples enfoques como una manera de verificar la consistencia de los resultados.

Los métodos directos incluyen mediciones de la concentración del agente en la zona de contacto entre el medio de exposición y el cuerpo humano. La recopilación de datos mediante el monitoreo personal o mediante el uso de marcadores biológicos de exposición se realiza en todos los individuos que participan en un estudio de DS. El monitoreo personal puede implementarse, en principio, para todos los medios de exposición (aire, agua, suelo, alimentos).

Los métodos indirectos incluyen el monitoreo de la contaminación en (micro) ubicaciones donde ocurre la exposición, el desarrollo de modelos de exposición individual o poblacional y la recopilación de información mediante métodos cualitativos como los cuestionarios exploratorios.

Entonces, para evaluar la exposición directa, hay que determinar la concentración del contaminante a la que están expuestas las personas, esta se expresa en dosis diaria de exposición y se determina con la ecuación (1).

$D D E_{i j}=C_{i} * Q_{j} * T * \frac{F}{P} * T m$

Donde:

$D D E_{i j}=$ dosis diaria de exposición relacionada con una exposición al medio i por vía de exposición $\mathrm{j}$ (en $\mathrm{mg} / \mathrm{kg} /$ día $)$
$C i=$ concentración de exposición relativa al medio i

$Q j=$ tasa de contacto, cantidad del medio suministrada por la vía $\mathrm{j}$ al día

$T=$ duración de exposición (años)

$F=$ frecuencia de exposición (días/año)

$P=$ peso corporal del afectado $(\mathrm{kg})$

$\mathrm{Tm}$ = periodo de tiempo en el cual se determina la cantidad media de exposición (días)

Para la vía respiratoria, se sustituye la dosis de exposición por la concentración inhalada ecuación (2).

$C I=\left[\sum\left(C_{i} * t_{i}\right)\right] * \frac{T * F}{T m}$

Donde:

$C I=$ Concentración inhalada

$C i=$ concentración de contaminante en el aire durante la fracción de tiempo $t i$

Para agentes cancerígenos y exposiciones crónicas, se calcula la dosis diaria vitalicia promedio (DDVP) ecuación (3).

$D D V P=C_{i} * Q_{j} * B * T * \frac{F}{P} * \operatorname{Tm}$

Donde:

$B=$ biodisponibildad (porcentaje que se ingiere o se absorbe) Se seleccionará la hipótesis de una homogeneidad de las concentraciones en el tiempo de exposición $t_{i}$.

Evaluación de la dosis-respuesta. Es la estimación de la relación matemática entre la magnitud de la exposición y la probabilidad de ocurrencia de efectos sobre la salud. Tiene en cuenta la intensidad de la exposición, el patrón de edad y otras variables que pudieran afectar a la respuesta, como sexo, estilos de vida y otros factores modificadores. Es decir, la dosis, además de las condiciones de exposición (vía de ingreso, duración y frecuencia de las exposiciones, tasa de contacto con el medio contaminado, etc.) determinan el tipo y magnitud de la respuesta biológica, la cual puede ser un efecto adverso.

Cabe señalar que, dependiendo de su naturaleza, cada agente tóxico induce efectos adversos mediante mecanismos fisiológicos y metabólicos diferentes, por lo que el tipo de efecto (cancerígeno y no-cancerígeno) que su exposición causa, determina la forma que adquiere la relación dosis-respuesta. De acuerdo a dicha relación, puede establecerse la existencia de una dosis a partir de la cual se presentan los efectos adversos (dosis umbral), la cual se presenta para los agentes no-cancerígenos, pero no así para los cancerígenos y que sirve para estimar el índice de toxicidad. 
Caracterización del riesgo. Es la parte final de la ER, así como del DS, la cual consiste en describir las principales características perjudiciales de cada riesgo, en caso de pasar del terreno de la probabilidad al de los hechos consumados. Es el resultado del análisis y resumen de las tres fases previas. Sánchez-Aguilar (2009) jerarquiza a los riesgos con base en los siguientes criterios: el mayor efecto nocivo, la menor DL50, condiciones particulares de la exposición (frecuencia, duración e intensidad), el grupo homogéneo de exposición y la probabilidad de ocurrencia.

Es importante diferenciar entre la caracterización del riesgo cancerígeno y el no cancerígeno. En este último, el índice de toxicidad utilizado es la dosis de referencia crónica (DdR), definido por la USEPA como el nivel de exposición diario de una población, durante toda la vida, para el que no existe un riesgo apreciable de efectos adversos. Por otro lado, el índice empleado para medir el riesgo asociado a la exposición a sustancias cancerígenas es el factor de pendiente para el cáncer, que presenta cuantitativamente la relación entre la dosis de agente cancerígeno y la incidencia de tumores, se emplea para calcular la probabilidad de desarrollar cáncer como resultado de una exposición a una sustancia (Taracido-Trunk 2011).

Existen bases de datos toxicológicos que permiten identificar valores de referencia aprovechables para una cuantificación del riesgo al que está sometida la población general. Entre ellas destacan el Sistema IRIS (de la USEPA, Sistema de información integral del riesgo, por sus siglas en inglés), las tablas HEAST (de la Agencia para Sustancias Tóxicas y el Registro de Enfermedades, ATSDR por sus siglas en inglés), así como valores guía que proporciona la OMS.

\section{HERRAMIENTAS}

Para mejorar la evaluación del impacto que los agentes tóxicos producidos en un SDF tienen sobre la salud de la población expuesta, es de utilidad la integración de información sobre el estado del sitio, el ecosistema y la población en riesgo potencial. Los sistemas de información geográfica (SIG) son una herramienta útil para realizar dicha integración y valorar el potencial y las restricciones naturales de uso de un sitio, para el desarrollo de una actividad específica y por consiguiente permiten visualizar espacialmente las amenazas ligadas a dichas actividades. Los SIG basados en el análisis de idoneidad amplían los enfoques tradicionales de los sitios a evaluar, al considerar características descriptivas (atributos) y datos espaciales. Una práctica común entre estos enfoques es que la evaluación de alternativas se produce en un contexto de decisión no espacial, donde el rendimiento de alternativas de sitios de ubicación, en los criterios de análisis ya se conoce (Demesouka et al. 2014).
En México, se puede obtener la cartografía digital (compatible con los software ArcGIS ${ }^{\circledR}$ para SIG), de manera gratuita y directamente de los portales oficiales de la Comisión Nacional para el conocimiento y uso de la Biodiversidad (CONABIO 2012), de la Comisión Nacional del Agua (CONAGUA 2012) y del Instituto Nacional de Estadística y Geografía (INEGI 2014). Esta disponibilidad de información facilita el desarrollo de modelos espaciales que integren información cartográfica al análisis de riesgo.

Con respecto al manejo de residuos, los SIG ya han sido utilizados para evaluar la localización de RESAs. Zamorano et al. (2008), Marín et al. (2012) y Gbanie et al. (2013) mencionan que la ubicación de RESAs debe tener en cuenta una amplia gama de factores territoriales y legales con el fin de reducir los impactos negativos sobre el medio ambiente y que debido a que es un proceso multidisciplinario complejo, requiere de una herramienta de análisis que abarque todos los factores involucrados y una gran cantidad de datos de varias fuentes que proyecte los resultados de manera sencilla.

Zamorano et al. (2008) presentan un método que identifica y clasifica las posibles áreas para la ubicación preliminar del sitio para la ciudad de Granada, España, con un arreglo para solucionar los problemas de múltiples criterios, éste método combina SIG con un sistema de toma de decisiones. Marín et al. (2012) utilizando SIG identificaron sitios posibles para la localización de un RESA en el estado de Morelos, México, con base en datos del SIGAMC (Sistema de Información Geográfico del Agua en México), una plataforma tecnológica cuya geobase de datos contiene información en los rubros de agua, suelo, infraestructura hídrica, y riesgos.

El desarrollo de métodos de cálculo con capacidad para modelar sistemas, permite cuantificar los peligros y sus efectos para simular y analizar con mayor precisión los riesgos que deben identificarse, lo que lleva a una gestión más eficaz del riesgo (Butt et al. 2008a). Existen modelos pertinentes, asistidos por computadora, con diversos enfoques que pueden apoyar como herramientas (cuantificación de emanaciones, capacidad de retención de humedad, ubicación geográfica del sitio, etc.) en la ER en SDF. Algunos de estos modelos son:

- MODUELO 4.0 (López-Martínez et al. 2012)

- Modelo Mexicano de Biogás (SCS-ENGINEERS y USEPA 2009)

- Modelo del Panel Intergubernamental de Cambio Climático (IPCC, por sus siglas en inglés) (IPCC 2007)

- LandSim (Environment Agency 2003)

- Método de balance de aguas (WBM, por sus siglas en inglés) (Fenn et al. 1975)

- Modelo de Evaluación del rendimiento hidrogeológico de un Vertedero (HELP, por sus siglas en inglés) (Scientific Software Group 1998) 
Diversos estudios de modelos matemáticos empleados para estimar la generación de biogás en RESAs han sido publicados (IPCC 2007; Aguilar-Virgen et al. 2011; Friedrich y Trois 2011; Kamalan et al. 2011), donde se dan a conocer varios modelos con diferentes órdenes de cinética, desde los más simples de orden cero, de primero y segundo orden, así como algunos modelos más complejos. Para nuestro país existe el Modelo Mexicano del Biogás (SCS-ENGINEERS y USEPA 2009), que ha sido utilizado por Cárdenas-Moreno (2014), para la estimación la generación de biogás en varios SDF del Estado de México (EM) y por García-Darás et al. (2013), quienes realizaron un análisis comparativo entre los modelos de generación de biogás aplicados a las emisiones de un relleno sanitario en Zapopan, Jalisco.

En lo referente a los lixiviados, hasta la fecha, muy pocos datos están disponibles sobre los volúmenes generados en los vertederos de RSU, sobre todo en los sitios donde el manejo no es adecuado. La mayoría de las estimaciones se basan en el enfoque de modelado y es difícil su validación en campo (Aziz et al. 2012). Una serie de herramientas se pueden emplear para predecir la generación y movilidad de lixiviados de SDF, como el Método de Balance de Agua (WBM, Water Balance Method), desarrollado por la Agencia de Protección Ambiental de E.U. (USEPA, por sus siglas en inglés) (Fenn et al. 1975), el modelo computacional para la Evaluación Hidrológica de Rendimiento del Vertedero (HELP, Hydrologic Evaluation of Landfill Performance) (Schroeder et al. 1994) y la investigación del flujo de lixiviados de vertederos (FILL, the Flow Investigation of Landfill Leachate) (Khanbilvardi et al. 1995). Todos ellos ampliamente utilizados para la estimación cuantitativa (pero no cualitativa) de lixiviados y que consideran tres factores críticos que son el almacenamiento de humedad del suelo, la evapotranspiración y la escorrentía superficial del agua. Además de estos modelos, más recientemente, se han desarrollado algunos algoritmos de predicción con técnicas redes neuronales artificiales (ANN), cuya aplicación ha dado resultados relativamente precisos (Chang y Wang 2009).

\section{ESTUDIOS DE CASO DE ER EN SDF}

En México se han realizado estudios de ER tanto a la salud como al ambiente, empleando diferentes metodologías. Por ejemplo González et al. (2002) aplicando la metodología de la USEPA realizaron un estudio de evaluación de riesgo a la salud de la población del área circundante a un SDF por consumo de agua contaminada por lixiviados provenientes del sitio en la ciudad de Mérida, Yucatán. Por otro lado, un estudio realizado por Vargas-Delgado (2015), muestra un análisis de las condiciones laborales de trabajadores de SDF de un RESA en el Estado de México, se trata de un estudio exploratorio, descriptivo observacional dividido en dos fases: el levantamiento de la información y valoraciones clínicas y estudios de laboratorio. Bernache-Pérez (2012) evaluó la situación de operación de 41 SDF de la Región Centro-Occidente de México (nueve estados de la república), y reporta que el $23 \%$ opera como Sitios No Controlados, $49 \%$ como Sitios Controlados y $28 \%$ como RESAs; además establece que los RESAs, al estar localizados, por lo general, en las ciudades de mayor tamaño y en las capitales de los estados, captan más de la mitad de los RSU de la región, mientras que los Sitios No Controlados tienden a ubicarse en los municipios de menor tamaño (por número de habitantes) ya que son los que tienen mayores dificultades presupuestales para construir y mantener obras de infraestructura complejas como las que requiere un RESA. Por su parte, Atencio-Pérez et al. (2013) evaluaron el riesgo ambiental y potencial en la salud por compuestos derivados de la quema de residuos dispuestos en un Sitio No Controlado en Mexicali, Baja California, entre los que se encontraron: metales, dioxinas (PCDD) y furanos (PCDF), bifenilos policlorados (PCB) y compuestos orgánicos semivolátiles; encontrando niveles que indican un riesgo alto (inaceptable) para los seres humanos en un escenario de uso residencial, con la mayoría de los riesgos cancerígenos atribuidos a la 2,3,7,8-tetraclorodibenzo-para-dioxina (TCDD), cuyas concentraciones en suelo variaron de 1.9 a $104 \mathrm{pg} / \mathrm{g}$. A partir de tales concentraciones, la exposición estimada en el escenario residencial resultó de alrededor de $1.04 \times 10^{-3}$ $\mathrm{mg} / \mathrm{kg}$ en adultos, la cual se encontró por arriba del valor de $1 \times 10^{-9} \mathrm{mg} / \mathrm{kg} /$ día correspondiente a la dosis de referencia oral (RFDo) de la TCDD (ORNL 2012).

\section{CONCLUSIONES}

En la actualidad no existe una metodología de análisis de riesgos de SDF basada en un sistema integrado y unificado que contemple la normatividad y los diferentes aspectos del riesgo en estos sitios (gestión de residuos, diseño del sitio, vulnerabilidad, etc.). Sin embargo, conjuntando los aspectos analizados en el presente trabajo se podría conocer de manera más completa el manejo de los sitios $\mathrm{y}$, utilizando algunos modelos asistidos por computadora como herramienta para estimar las emanaciones de los SDF, podría mejorarse el proceso de ER por dichas emanaciones. Con base en dichos aspectos podría establecerse una metodología preliminar para determinar la situación actual de un SDF. Asimismo, un primer estudio de posibles efectos dañinos a la salud causados por los contaminantes emitidos, se puede desarrollar tomando en cuenta al personal ocupacionalmente expuesto $y$, posteriormente ampliar 
la ER a poblaciones y medio ambiente cercanos, basada en un análisis multicriterio en el entorno de los SIG que permita la integración de criterios y condicionantes y la búsqueda de interrelaciones para la toma de decisiones acertadas en las áreas de la gestión de residuos, manejo del riesgo y la salud pública. Aún existe una serie de vacíos en la literatura revisada y establecer un sistema de línea de base integral de estudio podría ayudar a llenar esos vacíos.

Así pues, el diseño, construcción, operación, supervisión y control del emplazamiento de rellenos sanitarios representan factores que la autoridad ambiental tiene que vigilar en sus diferentes ámbitos de gobierno. Por lo anterior, se requieren estudios de riesgo e impacto ambiental ayudados por herramientas informáticas dinámicas como los SIG, que permitan la visualización de este tipo de obras a lo largo del tiempo, así como elaborar un modelo espacial integrado de información sobre los diversos factores que constituyen una amenaza para la salud de grupos poblacionales en la zona de impacto de los SDF de RSU, minimizando así los riesgos a la salud pública y al ambiente. Los resultados de un análisis multicriterio podrían fortalecer la toma de decisiones de las autoridades en acciones preventivas y de control en la gestión y manejo de SDF de RSU, en congruencia con programas de desarrollo urbano municipal para justificar la construcción de nuevos SDF, así como la posible reubicación y/o saneamiento de los ya existentes y que se encuentran en zonas de riesgo.

\section{AGRADECIMIENTOS}

Los autores agradecen al IPN-SIP (Proyecto 20161464) por el apoyo financiero que permitió el desarrollo de este proyecto.

\section{REFERENCIAS}

Aguilar-Virgen Q., Taboada-González P., Ojeda-Benitez S., y Cruz-Sotelo S. (2011). Modelo mexicano para la estimación de la generación de biogás. Ingenieria, 15(1), 37-45

Atencio-Pérez R. M., Reyes-López J. A., y GuevaraGarcía J. A. (2013). Evaluación de riesgo ambiental en un tiradero con quema de basura. Rev. Int. Contam. Ambie., 29(Sup. 3), 107-117

Aziz H. A., Adlan M. N., Amilin K., Yusoff M. S., Ramly N. H., y Umar M. (2012). Quantification of leachate generation rate from a semi-aerobic landfill in Malaysia. Environmental Engineering and Management Journal, 11(9), 1581-1585

Ballester-Díez F., Valcárcel-Rivera Y. y Lacasaña-Navarro M. (2011). Epidemiología Ambiental. En Manual de
Epidemiología y salud pública: para grados en ciencias de la salud (2da. ed.) (I. Hernández-Aguado, Ed.). Médica Panamericana, Madrid, España, pp. 229-233.

Bernache-Pérez G. (2012). Riesgo de contaminación por disposición final de residuos. Un estudio de la región centro Occidente de México. Rev. Int. Contam. Ambie., 28(Supl 1), 97-105

Buenrostro O., Márquez, L. y Pinette, F. (2008). Consuption patterns and household hazardous solid waste generation in an urban settlement in Mexico. Waste Management, 28, s2-s6. DOI: 10.1016/j.wasman. 2008.03.019

Butt T. E., Lockley E. y Oduyemi K. O. K. (2008a). Risk assessment of landfill disposal sites - State of the art. Waste Management, 28(6), 952-964.

DOI: $10.1016 /$ j.wasman.2007.05.012

Butt T. E., Davidson H. A. y Oduyemi K. O. K. (2008b). Hazard assessment of waste disposal sites. Part 2: a holistic approach for landfill leachates. International Journal of Risk Assessment and Management, 10(1/2), 109. http://doi.org/10.1504/IJRAM.2008.021057

Butt T. E., Gouda H. M., Baloch M. I., Paul P., Javadi A. y Alam A. (2013). Literature review of baseline study for risk analysis - The landfill leachate case, 63, 149-162. http://doi.org/10.1016/j.envint.2013.09.015

Cárdenas-Moreno P. R. (2014). Estimación de biogás y lixiviados generados en sitios de disposición final de residuos sólidos urbanos del Estado de México. Tesis de Maestría. Unidad Profesional Interdisciplinaria de Biotecnología - Instituto Politécnico Nacional, México

Chang S. y Yi Wang Y. (2009) Prediction of leachate flow-rate in a MSW landfill site using neural networks method. Journal of Solid Waste Technology \& Management. 35 (2), 104-114.

Chiemchaisri C. y Visvanathan, C. (2008). Greenhouse gas emission potential of the municipal solid waste disposal sites in Thailand. Journal of the Air \& Waste Management Association, 58, 629-635.

DOI: $10.3155 / 1047-3289.58 .5 .629$

Colomer-Mendoza F. J., Esteban-Altabella J., GarcíaDarás F., y Herrera-Prats L., Robles-Martínez, F. (2013a). Influencia de la ubicación de los rellenos sanitarios en el impacto ambiental. Caso de España. Ingeniería, 2(17), 141-151.[en línea] http://www. redalyc.org/articulo.oa?id $=46730913005$

Colomer-Mendoza F. J., García-Darás F., Vives-Peris V., y Robles-Martínez F. (2013b). Aspectos ambientales a considerar en vertederos: Emplazamiento y emisiones de biogás. DYNA, 88, 726-730. [en línea]. http://www. revistadyna.com/busqueda/aspectos-ambientales-a-considerar-en-vertederos-ubicacion-y-emisiones-de-biogas

Colomer-Mendoza F. J. y Robles-Martínez F. (2011). Generación de biogás en los vertederos controlados. Agrónomos, 41, 10-17 
CONABIO (2012). Portal de Geoinformación Sistema Nacionalde Información sobre Biodiversidad [en línea]. http://www.conabio.gob.mx/informacion/gis 24/04/2015

CONAGUA (2012). Atlas digital del agua México 2012 [en línea]. http://siga.cna.gob.mx/ 16/05/2015

Davoli E., Fattore E., Paiano V., Colombo A., Palmiotto M., Rossi A. N. y Fanelli R. (2010). Waste management health risk assessment: A case study of a solid waste landfill in South Italy. Waste Management, 30 (8-9), 1608-1613.

DOI: $10.1016 /$ j. wasman.2009.10.013

Del Pozo-Manrique M. (1991). Selección de emplazamientos para vertederos controlados. Informes de la Construcción, 42(412), 23-40.

DOI: $10.3989 /$ ic. $1991 . v 42.1412 .1393$

Demesouka O. E., Vavatsikos A. P., y Anagnostopoulos K. P. (2014). GIS-based multicriteria municipal solid waste landfill suitability analysis: a review of the methodologies performed and criteria implemented. Waste Management and Research, 32(4), 270-296. http://doi. org/10.1177/0734242X14526632

Environment Agency (2003). LIT 1773. LandSim 2.5 Groundwater risk from assessment tool for landfill design. Bristol.

Environment Agency (2010). P1-396/R. Exposure Assessment of Landfill Sites Volume 1: Main report (Vol. 1)

Fenn, D., Hanley, K. y DeGeare, T. (1975). SW-168. Use of the water-balance method for predicting leachate generation from solid-waste-disposal sites. Cincinnati, Ohio.

Forastiere F., Badaloni C., de Hoogh K., von Kraus M. K., Martuzzi M., Mitis F. y Briggs D. (2011). Health impact assessment of waste management facilities in three European countries. Environmental Health: A Global Access Science Source, 10(1), 53. http://doi. org/10.1186/1476-069X-10-53

Friedrich E. y Trois C. (2011). Quantification of greenhouse gas emissions from waste management processes for municipalities - A comparative review focusing on Africa. Waste Management, 31(7), 1585-1596. http:// doi.org/10.1016/j.wasman.2011.02.028

García-Darás F., Colomer M. F. J., Robles-Martínez F. y Aranda G. (2013). Análisis comparativo entre los modelos de generación de biogás aplicados a las emisiones de un relleno sanitario en México. Memorias. V Simposio Iberoamericano de Ingeniería de Residuos (p. 6). Mendoza, Argentina. 15 y 16 de octubre de 2013. ISBN: 978-987-1323-35-7

Gbanie S. P., Tengbe P. B., Momoh J. S., Medo J. y Kabba V. T. S. (2013). Modelling landfill location using Geographic Information Systems (GIS) and Multi-Criteria Decision Analysis (MCDA): Case study Bo, Southern
Sierra Leone. Applied Geography, 36, 3-12. http://doi. org/10.1016/j.apgeog.2012.06.013

González R., Casarez R. y Rodríguez R. (2002). Riesgo a la salud por consumo de agua contaminada por lixiviados de basureros. Memorias. XXVIII Congreso de Ingeniería sanitaria y ambiental, Cancún, México 2002 (pp. 1-8). Cancún. [en línea] http://www.bvsde.paho. org/bvsacd/agua2003/lixi.pdf 29/03/2016

González C., Buenrostro O., Márquez L., HernándezC., Moreno, E. y Robles F. (2011). Effect of Solid Wastes Composition and Confinement Time on Methane Production in a Dump. Journal of Environmental Protection, 02(10), 1310-1316. http://doi.org/10.4236/ jep.2011.210151

IMSS (2014). Instituto Mexicano del Seguro Social. Memoria Estadística 2014, Capítulo VI Salud en el trabajo [en línea]. http://imss.gob.mx/conoce-al-imss/ memoria-estadistica-2014 05/08/2016

INEGI (2012). Residuos Sólidos Urbanos. Censo Nacional de Gobiernos Municipales y Delegacionales 2011 [en línea]. http://www3.inegi.org.mx/sistemas/temas/ default.aspx?s=est\&c=21385 05/05/2015

INEGI (2014). Geografía [en línea]. http://www.inegi.org. $\mathrm{mx} /$ geo/contenidos/recnat/default.aspx 05/05/2015

IPCC (2007). Eliminación de desechos sólidos. En R. Pipatti y P. Svarda (Eds.), Directrices del IPCC de 2006 para los inventarios nacionales de gases de efecto invernadero (p. 44). Ginebra, Suiza.

Isidori M., Lavorgna M., Nardelli A. y Parrella A. (2003). Toxicity identification evaluation of leachates from municipal solid waste landfills: a multispecies approach. Chemosphere, 52(1), 85-94. http://doi. org/10.1016/S0045-6535(03)00298-4

Kamalan H., Sabour M. y Shariatmadari N. (2011). A review on available landfill gas models. Journal of Environmental Science and Technology. 4(2), 79-92. http://doi.org/10.3923/jest.2011.79.92

Khanbilvardi R. M., Ahmed S. y Gleason P. J. (1995). Flow investigation for landfill leachate (FILL), Journal of Environmental Engineering. 121(1), 45-57.

Kiss-Köfalusi G. y Encarnación-Aguilar G. (2006). Los productos y los impactos de la descomposición de residuos sólidos urbanos en los sitios de disposición final. Gaceta Ecológica, 79, 39-51. ISSN:1405-2849

Kloppenborg S. C., Brandt U. K., Gulis G. y Ejstrud B. (2005). Risk of congenital anomalies in the vicinity of waste landfills in Denmark; an epidemiological study using GIS. Central European Journal of Public Health, 13(3), 137-143. [en línea] http://apps.szu.cz/svi/cejph/ archiv/2005-3-06-full.pdf 15/08/2015

López-Martínez A., Gonzalorena-Vallejos R. J., CuartasHernández M. y Lobo-García de Cortázar, A. (2012). Modelación de una celda de vertedero experimental 
con MODUELO 4.0. Rev. Int. Contam. Ambie., 28(1), 89-96.

Mancheño M., Izquierdo M., Martin A., García A., Gutierrez A., Peinado A. y Heras M. (2008). Exposición laboral a disolventes (1 ra. ed.). Unión sindical de Madrid región de CC.OO. Madrid, España. pp 228.

Marín L. E., Torres V., Bolongaro A., Reyna J. A., Pohle O., Hernández-Espriú A. y Tabla H. F. P. (2012). Identifying suitable sanitary landfill locations in the state of Morelos, México, using a Geographic Information System. Physics and Chemistry of the Earth, 37-39, 2-9. http://doi.org/10.1016/j.pce.2011.12.003

Martínez-Ponce de León J. G. (2007). Introducción al análisis de riesgos (1 ra. ed.). Limusa - Noriega editores. México. ISBN 968-18-6153-1

NIOSH (2012). Bases de datos-hojas de seguridad [en línea]. http://www.cdc.gov/spanish/niosh/db-sp.html 22/06/2015

Öman C. B. y Junestedt C. (2008). Chemical characterization of landfill leachates - 400 parameters and compounds. Waste Management, 28(10), 1876-1891. http://doi.org/10.1016/j.wasman.2007.06.018

OMS (2001). EUT/00/i5026441. Métodos de evaluación de riesgos para la salud generados por la exposición a sustancias peligrosas liberadas por rellenos sanitarios. Informe de una Reunión de la OMS LODZ, Lodz, Polonia.

OMS (2014). Estadísticas Sanitarias Mundiales [en línea]. http://www.who.int/gho/publications/world_health_ statistics/2014/es 29/06/2015

ORNL (2012). Toxicity values. Risk Assess. Inf. Syst. [en línea] https://rais.ornl.gov/tutorials/toxvals.html 08/01/2016

Ortiz-Morales I. C. (2010). Diagnóstico situacional sobre el manejo de los residuos peligrosos biológico infecciosos (RPBI) en el personal de intendencia de un Centro de Salud TIII de la ciudad de México. Tesis de Maestría. Escuela Nacional de Medicina y Homeopatía, Instituto Politécnico Nacional. [en línea] http://www. enmh.ipn.mx/PosgradoInvestigacion/Documents/ tesismsosh/ORTIZMORALESIGNACIOCARLOS. pdf 25/09/2015

OSHA (2012). Fichas de Datos de Seguridad para la Comunicación de Peligro [en línea]. https://www.osha. gov/Publications/HazComm_QuickCard_SafetyDataSpanish.html 29/06/2015

Palmiotto M., Fattore E., Paiano V., Celeste G., Colombo A. y Davoli E. (2014). Influence of a municipal solid waste landfill in the surrounding environment: Toxicological risk and odor nuisance effects. Environment International, 68, 16-24. http://doi.org/10.1016/j. envint.2014.03.004
Porta D., Milani S., Lazzarino A. I., Perucci C. A. y Forastiere F. (2009). Systematic review of epidemiological studies on health effects associated with management of solid waste. Environmental Health: A Global Access Science Source, 8, 60. http://doi.org/10.1186/1476069X-8-60

Pukkala E. (2014). A follow-up of cancer incidence among former Finnish dump site residents: 1999-2011. International Journal of Occupational and Environmental Health, 20(4), 313-317. http://doi.org/10.1179/20493 96714Y.0000000080

Robles-Martínez F. (2008). Generación de biogás y lixiviados en los rellenos sanitarios (2da. ed.). Instituto Politécnico Nacional. Distrito Federal, México

Robles-Martínez F., Morales-López Y., Piña-Guzmán A. B., Espíndola-Serafín O., Tovar-Gálvez L. R. y Valencia-del Toro G. (2011). Medición de pH y cuantificación de metales pesados en los lixiviados del relleno sanitario más grande de la zona metropolitana de la ciudad de méxico. Univ. y Cienc. 27, 121-132

Rojas-Alegría A. M. (2011). Diagnóstico situacional para riesgos químicos en laboratorios de investigación y propuesta de atención y prevención. Tesis de Maestría. Escuela Nacional de Medicina y Homeopatía, Instituto Politécnico Nacional. Distrito Federeral, México

Sánchez-Aguilar M. (2009). Diseño de un método de diagnóstico integral para detección y control de enfermedades laborales en una empresa refresquera. Tesis de Maestría. Escuela Nacional de Medicina y Homeopatía, Instituto Politécnico Nacional. Distrito Federeral, México.

Sánchez-Aguilar M. (2013). Diseño de un método de diagnóstico integral de enfermedades derivadas de los riesgos laborales presentes en procesos productivos. Tesis de Doctorado. Escuela Superior de Ingeniería Mecánica y Eléctrica, Instituto Politécnico Nacional. Distrito Federeral, México.

Schroeder P. R., Aziz N. M., Lloyd C. M. y Zappi P. A. (1994). The Hydrologic Evaluation of Landfill Performance (HELP) Model: User's Guide for Version 3, EPA/600/R-94/168a, September 1994, U.S. Environmental Protection Agency Office of Research and Development, Washington, DC.

Scientific Software Group (1998). Environmental Software and Publications (Catalogue). Washington, DC.

SCS-ENGINEERS y USEPA (2009). Manual del Usuario Modelo Mexicano de Biogás, Versión 2.0. EPA Landfill Methane Outreach Program. [en línea] http://www. epa.gov/lmop/international/mexicano.html 29/09/2015

SEDEMA (2006). Gestión ambiental del aire en el Distrito Federal 2006-2011. 1ra. Edición. Secretaría de Medio Ambiente del Distrito Federal. México, 271 pp. 
SEMARNAT (2003). NOM-083-SEMARNAT-2003, Especificaciones de protección ambiental para la selección del sitio, diseño, construcción, operación, monitoreo, clausura y obras complementarias de un sitio de disposición final de residuos sólidos urbanos y de manejo especial. Diario Oficial de la Federación. 20 de octubre de 2004

SEMARNAT (2005). NOM-052-SEMARNAT-2005, Que establece las características, el procedimiento de identificación, clasificación y los listados de los residuos peligrosos. Diario Oficial de La Federación. 23 de junio de 2006

Sissinno C. L. S., Oliveira-Filho E. C., Dufrayer M. C., Moreira J. C. y Paumgartten F. J. R. (2000). Toxicity evaluation of a municipal dump leachate using zebrafish acute tests. Bulletin of Environmental Contamination and Toxicology, 64(1), 107-113. http://doi. org/10.1007/s001289910017

Soltani-Ahmadi H. (2000). A review of the literature regarding non-methane and volatile organic compounds in municipal solid waste landfill gas. Newark, Delaware: University of Delaware: SWANA/Hickman Intern.

Spokas K., Bogner J., Chanton J. P., Morcet M., Aran C., Graff C. y Hebe I. (2006). Methane mass balance at three landfill sites: What is the efficiency of capture by gas collection systems? Waste Management, 26(5), 516-525. http://doi.org/10.1016/j.wasman.2005.07.021

Taracido-Trunk M. (2011). Evaluación de riesgos ambientales, En: Manual de epidemiología y salud pública para grados en ciencias de la salud (2a. ed.,) (I. Hernández-Aguado. Ed.). Médica Panamericana, Madird, España. pp. 241-244.
Themelis N. J. y Ulloa P. A. (2007). Methane generation in landfills. Renewable Energy, 32(7), 1243-1257. http:// doi.org/10.1016/j.renene.2006.04.020

Triassi M., Alfano R., Illario M., Nardone A., Caporale O. y Montuori P. (2015). Environmental pollution from illegal waste disposal and health effects: A review on the "triangle of death." International Journal of Environmental Research and Public Health, 12(2), 1216-1236. http://doi.org/10.3390/ijerph120201216

Vargas-Delgado J. F. (2015). Estudio y análisis de las condiciones laborales de los segregadores de residuos sólidos en un relleno sanitario de la ZMVM. Tesis de Maestría. Escuela Nacional de Medicina y Homeopatía, Instituto Politécnico Nacional. Distrito Federeral, México.

Vioque-López J., García-De la Hera M. y NavarreteMuñoz E. M. (2011). Principales causas de muerte y de enfermedad. En: Manual de epidemiología y salud pública para grados en ciencias de la salud (2a. ed.,) (I. Hernández-Aguado, Ed.). Médica Panamericana, Madrid, España, pp. 107-112.

Vrijheid M., Dolk H., Armstrong B., Boschi G., Busby A., Jorgensen T. y Pointer P. (2002). Hazard potential ranking of hazardous waste landfill sites and risk of congenital anomalies. Occupational and Environmental Medicine, 59, 768-776. http://doi.org/10.1136/ oem.59.11.768

Zamorano M., Molero E., Hurtado A., Grindlay A. y Ramos A. (2008). Evaluation of a municipal landfill site in Southern Spain with GIS-aided methodology. Journal of Hazardous Materials, 160(2-3), 473-481. http://doi.org/10.1016/j.jhazmat.2008.03.023 